\section{The Rediscovery of Hydroxychloroquine in Allergic Diseases in the COVID-19 Era}

Bonzano L ${ }^{1}$, Cassone $\mathrm{G}^{2,3}$, Tarallo $\mathrm{L}^{4}$, Pellacani $\mathrm{G}^{5}$

${ }^{1}$ Allergology Service, AUSL Policlinico of Modena, Modena, Italy

${ }^{2}$ Rheumatology Unit, University of Modena and Reggio Emilia,

Azienda Ospedaliero-Universitaria Policlinico di Modena,

Modena, Italy

${ }^{3}$ Rheumatology Unit, IRCCS Arcispedale Santa Maria Nuova, Azienda Unità Sanitaria Locale, Reggio Emilia, Italy

${ }^{4}$ Department of Orthopedic Surgery, University of Modena and Reggio Emilia, Azienda Ospedaliero-Universitaria Policlinico di Modena, Modena, Italy

${ }^{5}$ Dermatology Unit, University of Modena and Reggio Emilia, Modena, Italy

J Investig Allergol Clin Immunol 2021; Vol. 31(1): 85-86 doi: 10.18176/jiaci.0575

Key words: COVID-19. Hydroxychloroquine. Allergic diseases.

Palabras clave: COVID-19. Hidroxicloroquina. Enfermedades alérgicas.

\section{To the Editor:}

The outbreak of coronavirus disease 2019 (COVID-19) in northern Italy has not only radically changed our everyday life and organization of health services, but also forced us to rely on various classes of old and new drugs to contain SARS-CoV-2 symptoms. My colleagues and I work at the Policlinico Hospital, in Modena, Emilia-Romagna, the region with the second highest number of COVID-19 cases in Italy. The Italian National Institute of Health reported 168941 laboratory-confirmed cases in Italy on April 16, and of these, 21486 were in Emilia-Romagna.

Given the lack of specific antiviral therapies against COVID-19, current treatments focus mainly on symptomatic and respiratory support, even though several compounds, such as hydroxychloroquine, are being investigated for the treatment of this life-threatening disease. The only indication for hydroxychloroquine to date has been as prophylaxis in selected cases at high risk of infection [1].

We report the case of a 35-year-old man (normal weight, nonsmoker) with a 5-year history of severe seasonal rhinoconjunctivitis caused by pollen from trees (cypress, plane, poplar, birch), weed (pellitory), and grass that was associated with moderate asthma. The patient habitually experiences severe episodes of asthma and rhinoconjunctivitis and dramatic worsening of respiratory symptoms in early spring when working in watermelon greenhouses, which are infested with pellitory, thus severely limiting his professional activity and necessitating systemic medication to attenuate symptoms (cycles of oral corticosteroids). On some occasions in the past, serious worsening of the clinical condition forced the patient to seek assistance in the emergency department, where systemic corticosteroids and other reliever drugs were administered to control severe episodes of bronchial asthma.

During the pandemic, the patient continued working with watermelons, his main occupation, without changing his routine, because agriculture in Italy was not suspended during the lockdown. As a result, he reported sneezing, itching, and tearing (Rhinitis Control Scoring System [RCSS], 14) and dry cough and wheezing (Asthma Control Test [ACT], 10) associated with poor quality of life (Rhino-conjunctivitis Quality of Life Questionnaire [RQLQ], 4.6; Asthma Quality of Life Questionnaire [AQLQ], 2.1). The patient had started therapy for seasonal allergic rhinoconjunctivitis (bilastine $40 \mathrm{mg} / \mathrm{d}$, mometasone furoate 2 sprays/d) and asthma (beclomethasone/formoterol 100/6 $\mu \mathrm{g} 4 \mathrm{puffs} / \mathrm{d}$, montelukast $10 \mathrm{mg} / \mathrm{d}$, and short-course oral corticosteroids as needed).

Two weeks after initiating this regimen, the patient received prophylaxis with hydroxychloroquine $400 \mathrm{mg} / \mathrm{d}$ for 2 months, because a cohabiting family member was affected by COVID-19. He reported rapid and progressive improvement of allergy-related symptoms and quality of life (after 4 weeks of hydroxychloroquine: RCSS, 1; ACT, 25; RQLQ, 0.2;AQLQ, 6), which was sufficient for him to stop therapy with nasal corticosteroids and antihistamines, inhaled corticosteroids/longacting $\beta_{2}$ agonists, and leukotriene receptor antagonists without experiencing symptoms even when exposed to pellitory and grass pollens. We prescribed low-dose budesonide/formoterol as needed to prevent exacerbations; however, the patient never used his reliever therapy [2].

Surprisingly, the patient did not experience and continues to be free of allergy symptoms during therapy with hydroxychloroquine, even though he has suspended antiallergic therapies and it has been sunny and slightly windy with a high pollen rate in recent weeks. Despite the suspension of pulmonary function tests because of the reorganization of health care management during the pandemic, the significant clinical improvement prompted us to investigate the biological effects of hydroxychloroquine on allergic diseases. Few studies have evaluated the effectiveness of hydroxychloroquine in the treatment of inflammatory processes in which mast cells play a key role, as is the case of allergic diseases.

Our investigation begins with a pioneering study from 1989, where it was shown that the pretreatment of rat mast cells with chloroquine $(10-1000 \mu \mathrm{mol} / \mathrm{L})$ reduced their ability to degranulate and produce prostaglandin D2, resulting in the inhibition of phospholipase A2 and histamine activity [3]. Moreover, it has been shown in vitro that prolonged treatment with hydroxychloroquine for 3-5 weeks on human mast cells decreases the intracellular expression 
level of CD63 and modifies the expression pattern of CD63. Thus, hydroxychloroquine does not alter intracellular levels of tryptase and chymase, as detected by flow cytometry, but dramatically decreases the enzymatic activity of tryptase and the expression of key mast cell mediators, such as IL-8 and granulocyte-macrophage colony-stimulating factor [4]. These results were also confirmed by Charous et al [5], who reported the antiallergic effects of hydroxychloroquine in terms of selective and profound inhibition of $\mathrm{IgE}$ and improved airflow in patients with moderate symptomatic asthma. In fact, antimalarials seem to inhibit IL-4-driven synthesis of IgE by human peripheral blood lymphocytes, suggesting that hydroxychloroquine may decrease bronchial inflammation by reducing the $\mathrm{IgE}$ involved in its pathogenesis. Blocking histamine has been shown to induce bronchoconstriction and reproducibly decreased antigen-induced bronchoconstriction in animal models, suggesting potential utility in the treatment of asthma [5].

In light of these clinical observations and the potential immunopathological action of hydroxychloroquine, as well as its tolerability, rare toxicity, low cost, and immunomodulatory properties, it makes sense to investigate the potential role of this agent in the control of severe allergic diseases in selected cases. Prospective clinical trials are needed to further evaluate the use of hydroxychloroquine in this challenging field.

\section{Funding}

The authors declare that no funding was received for the present study.

\section{Conflicts of Interest}

The authors declare that they have no conflicts of interest.

\section{References}

1. Spinelli FR, Ceccarelli F, Di Franco M, Conti F. To consider or not antimalarials as a prophylactic intervention in the SARS-CoV-2 (Covid-19) pandemic. Ann Rheum Dis. 2020;annrheumdis-2020-217367.

2. www.ginasthma.org; update 2020.

3. Green KB, Lim HW. Effects of Chloroquine on Release of Mediators From Mast Cells. Skin Pharmacol. 1989;2(2):7785.

4. Espinosa E, Valitutti S, Laroche M, Laurent C, Apoil PA, Hermine 0 , et al. Hydroxychloroquine as a novel therapeutic approach in mast cell activation diseases. Clin Immunol. 2018;194:759.

5. Charous BL, Halpern EF, Steven GC. Hydroxychloroquine improves airflow and lowers circulating lgE levels in subjects with moderate symptomatic asthma. J Allergy Clin Immunol. 1998;102(2):198-203.

- Manuscript received April 29, 2020; accepted for publication May 28, 2020.

\section{Laura Bonzano}

Allergology Service, Policlinico of Modena

via del Pozzo, 71

41100 Modena, Italy

E-mail: laurabonzano83@gmail.com 